\title{
Sub-pixel porosity revealed by $x$-ray scatter dark field imaging
}

\author{
V. Revol, ${ }^{1,2, a)}$ I. Jerjen, ${ }^{3}$ C. Kottler, ${ }^{1}$ P. Schütz, ${ }^{3}$ R. Kaufmann, ${ }^{1}$ T. Lüthi, ${ }^{3}$ U. Sennhauser,${ }^{3}$ \\ U. Straumann, ${ }^{2}$ and C. Urban ${ }^{1}$ \\ ${ }^{1}$ Photonics Division, Centre Suisse d'Electronique et Microtechnique SA, Technoparkstr. 1, 8005 Zürich, \\ Switzerland \\ ${ }^{2}$ Physics Institute, University of Zürich, Winterthurerstr. 190, 8057 Zürich, Switzerland \\ ${ }^{3}$ Swiss Federal Laboratories for Materials Science and Technology (Empa), Überlandstr. 129, 8600 \\ Dübendorf, Switzerland
}

(Received 4 April 2011; accepted 28 June 2011; published online 31 August 2011)

\begin{abstract}
X-ray scatter dark field imaging based on the Talbot-Lau interferometer allows for the measurement of ultra-small angle x-ray scattering. The latter is related to the variations in the electron density in the sample at the sub- and micron-scale. Therefore, information on features of the object below the detector resolution can be revealed.

In this article, it is demonstrated that scatter dark field imaging is particularly adapted to the study of a material's porosity. An interferometer, optimized for x-ray energies around $50 \mathrm{keV}$, enables the investigation of aluminum welding with conventional laboratory $\mathrm{x}$-ray tubes. The results show an unprecedented contrast between the pool and the aluminum workpiece. Our conclusions are confirmed due to micro-tomographic three-dimensional reconstructions of the same object with a microscopic resolution. (C) 2011 American Institute of Physics. [doi:10.1063/1.3624592]
\end{abstract}

\section{INTRODUCTION}

$\mathrm{X}$-ray imaging is a standard tool for the non-destructive evaluation of manufactured objects. The currently implemented systems rely upon the measurement of the attenuation of the X-ray beam within the sample. Although such absorption-based equipment performs well in most applications, it is limited in detecting small inhomogeneities in a material. Magnification can be realized to achieve spatial resolutions on the order of a micrometer. However, the reconstructed volume is accordingly reduced and prevents the investigation of a large specimen within a reasonable measurement time.

The microstructure of manufactured objects can be of paramount importance. For example, porosity is a challenging issue in the injection molding of plastics and for metal welding. It impacts the aging properties of the materials, for example, the formation of cracks.

Recently, a method called scatter dark field imaging (SDFI) was demonstrated based on the Talbot-Lau interferometer, ${ }^{1-14}$ which relies on the measurement of the ultra-small angle x-ray scattering (USAS) caused by an inhomogeneous object. ${ }^{5}$ Since USAS is related to the variations in the electron density in the sample at the sub- and micron-scale, ${ }^{6}$ SDFI allows access to information on the sample below the resolution of the x-ray detector.

Furthermore, SDFI is a good candidate for industrial implementation. Indeed, this technique is compatible with conventional $\mathrm{x}$-ray sources ${ }^{4}$ and large field-of-view detectors, ${ }^{7}$ and can be combined with computed tomography ${ }^{8}$ and scanning schemes. ${ }^{9}$

This paper explains how SDFI can be implemented for the inspection of porosity at a sub-pixel length scale. A gra-

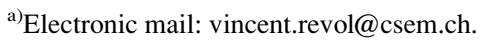

ting interferometer is presented that is optimized for $\mathrm{x}$-ray energies around $50 \mathrm{keV}$. It enables objects made out of light metals to be inspected with SDFI. It is shown that the spatial distribution of the pores can be recovered. Our assumptions are confirmed by high-resolution three-dimensional microcomputed tomography.

\section{SCATTER DARK FIELD IMAGING}

Scatter dark field imaging is based on the Talbot-Lau interferometer, which has been extensively documented in the literature. ${ }^{1-4,10-12}$ The latter consists of three $\mathrm{x}$-ray gratings, $G_{0}, G_{1}$, and $G_{2}$, added to a conventional x-ray tube source and detector as displayed in Fig. 1(a). The sample placed between $G_{0}$ and $G_{1}$ can be rotated around the $y$-axis in order to achieve computed tomography (CT).

As illustrated in Fig. 1(b), the phase grating, $G_{1}$, induces phase modulations of the x-ray wavefront, which cause interference fringes, $I_{f r}$, on the plane of $G_{2}$, at a distance, $d$, downstream of $G_{1}$. The intensity, $I_{m, n}$, recorded by the detector pixel $(m, n)$ ( $m$ and $n$ are integers identifying the pixel) varies as a function of the position, $\chi$, of the absorbing grating, $G_{2}$, with respect to $I_{f r}$ along the $x$-direction. The resulting curve, $I_{m, n}(\chi)$, called the phase stepping curve, forms a periodic function of period, $p_{2}$. Any change in the mean, amplitude, or position of the intensity pattern, $I_{f r}$, will thus appear in the phase stepping curve. The phase stepping method allows for the reconstruction of the absorption-based radiography and the SDFI signal. ${ }^{3}$

In particular, the SDFI signal, $V$, is given by the visibility, $v^{s}$, normalized by the visibility, $v^{r}$, when no sample is in the field of view. When the Talbot-Lau interferometer is used with broadband radiation, the visibility, $v(m, n)$, is equal to the ratio of the amplitude to the mean of the oscillating phase stepping curve, $I_{m, n}(\chi)$, as reported in Ref. 5. Thus, 
(a)

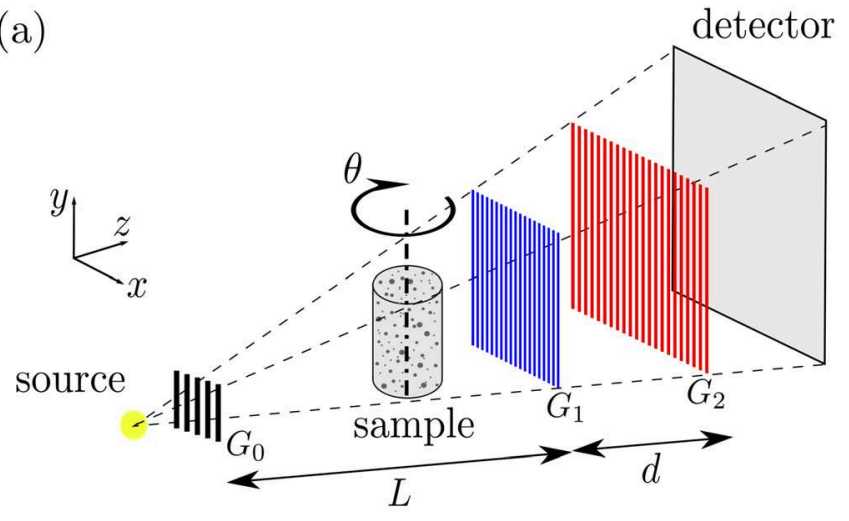

(b)

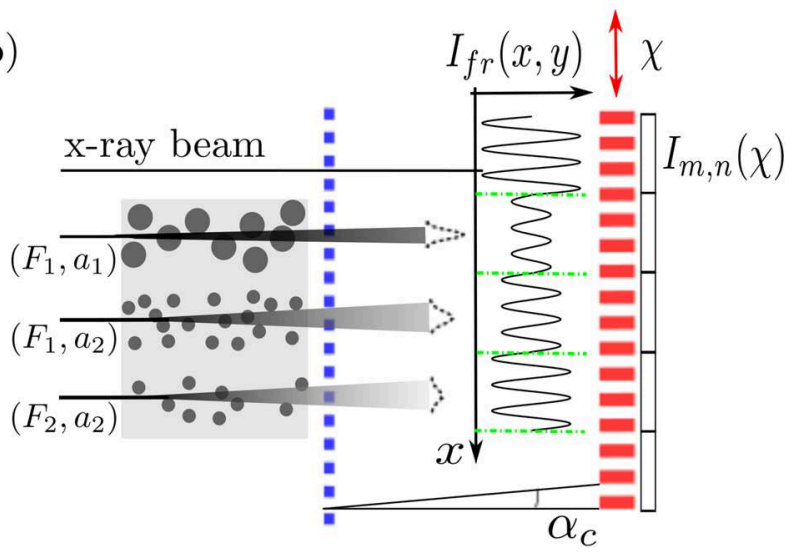

FIG. 1. (a) (Color online) Schematic view of the grating interferometer with x-ray source and detector and three x-ray gratings, $G_{0}, G_{1}$, and $G_{2} . L$ and $d$ are the distances between $G_{0}$ and $G_{1}$, and $G_{1}$ and $G_{2}$, respectively. The sample can be rotated around the $y$-axis by an angle, $\theta$. (Adapted from Ref. 13, Fig. 1). (b) A pure phase object composed of spherical inhomogeneities embedded in a uniform medium is placed in the x-ray beam. The interference pattern, $I_{f r}$, induced by $G_{1}$ onto the plane of $G_{2}$ is blurred due to the ultrasmall angle scattering. The decrease of the visibility (amplitude divided by the mean) of $I_{f r}$ varies with the size, $a$, and the volume fraction, $F$, of the particles as discussed in the text. The drawings are not to scale.

$v(m, n)$ is directly related to the contrast of the interference pattern, $I_{f r}$.

Let us consider now a pure phase object (meaning not absorbing) composed of spheres of material A embedded in material B, which are smaller than the pixel size. In the case of porous materials, A would be, for example, air. The propagation of the $\mathrm{x}$-ray beam through those multiple spheres randomly positioned results in variations of the wavefront, $\Phi_{f}$, which are fast compared to the pixel size. These unresolved variations lead to a broadening of the beam, as shown in Fig. 1(b), or equivalently, a loss of coherence of the radiation. This finally results in a blurring of the interference fringes, hence a decrease in the visibility, $v^{s}$.

Yashiro et al. ${ }^{6}$ developed theoretical expressions for the SDFI signal in the case of a monochromatic beam of wavelength, $\lambda$. Assuming that the ensemble of the phase signals, $\Phi_{f}$, over one pixel follows a Gaussian distribution, the SDFI signal can be expressed as, ${ }^{6}$

$$
V(m, n)=\exp \left(-\sigma^{2}(m, n)\left(1-\gamma\left(-N p_{1} ; m, n\right)\right)\right),
$$

where $\sigma$ is the width and $\gamma$ is the normalized autocorrelation function in the $x$-direction of the Gaussian distribution, $\Phi_{f}$.
Here, $p_{1}$ and $p_{2}$ are the periodicities of gratings, $G_{1}$ and $G_{2}$, while $N$ is the Talbot order defined as $N=2 d / d_{T}$ with $d_{T}=2 p_{1} p_{2} / \lambda$. Clearly, $V$ decreases with the width, $\sigma$, and is weighted by the autocorrelation of the phase variations over a length, $N p_{1}$. Perfectly uncorrelated phase variations $(\gamma=0)$ contribute fully to the visibility loss while correlated phase variations (in the extreme case, a uniform object) do not affect the signal, $V$.

For spherical inhomogeneities of radius, $a$, the quantities, $\sigma$ and $\gamma$, can be calculated analytically and are given by, ${ }^{6}$

$$
\begin{gathered}
\sigma^{2}=(F t) \cdot\left(\frac{3}{2} a \Delta \rho^{2} r_{e}^{2} \lambda^{2}\right), \\
\gamma\left(-N p_{1}\right)=\left(1+\frac{R^{2}}{2}\right) \sqrt{1-R^{2}} \\
+\left(2 R^{2}-\frac{R^{4}}{2}\right) \log \left(\frac{|R|}{1+\sqrt{1-R^{2}}}\right), \\
\text { with } R=\frac{N p_{1}}{2 a},
\end{gathered}
$$

where $F$ is the volume fraction occupied by the spherical inclusions (equal to the number density multiplied by the volume of a single sphere), $t$ is the thickness of the sample along the beam propagation, $r_{e}$ is the classical electron radius, and $\Delta \rho$ is the difference in the number density of electrons between materials $\mathrm{A}$ and $\mathrm{B}$.
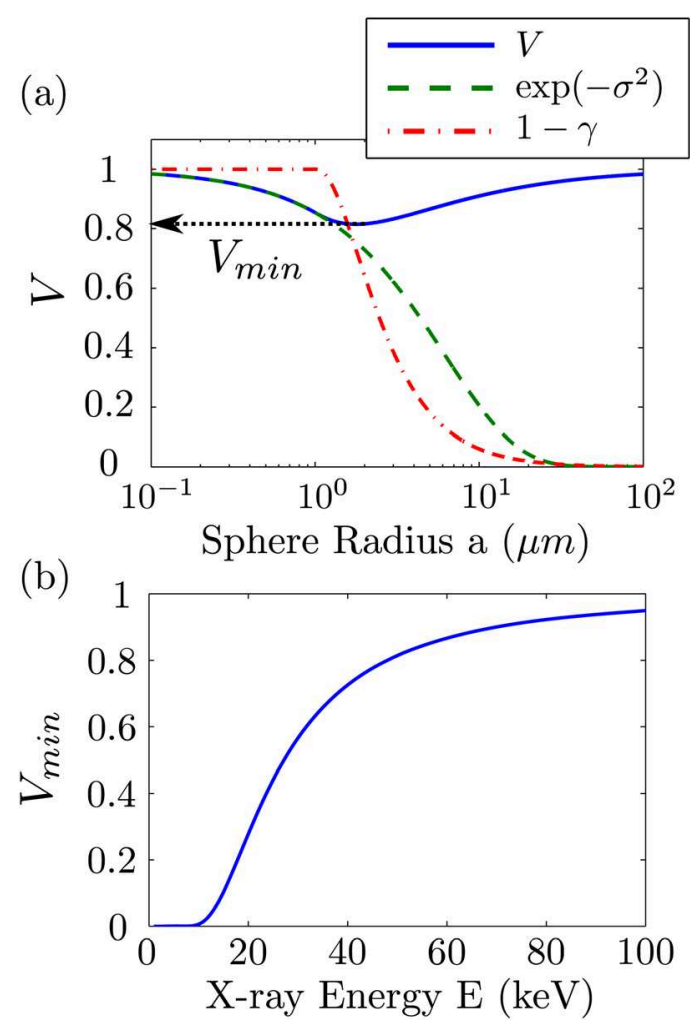

FIG. 2. (Color online) (a) Calculated SDFI signal for spherical air pockets trapped in an aluminum piece as a function of the radius for an x-ray energy of $50 \mathrm{keV}$. The other parameters were fixed at values, $p_{1}=4 \mu \mathrm{m}, N=1 / 2$, $F=0.5, t=10 \mathrm{~mm}$, and $\Delta \rho=66 \times 10^{29} \mathrm{~m}^{-3}$. (b) Minimum SDFI signal as a function of the x-ray energy. 
The quantity, $\sigma^{2}$, is linked to the intensity of the ultrasmall angle scattering. For the same volume fraction, the latter increases with the radius of the spherical inhomogeneities (green dotted line in Fig. 2(a)). On the other hand, the factor $(1-\gamma)$ is associated with the ratio of the scattering angle, $\alpha$, over the critical angle of the interferometer, $\alpha_{c}=p_{2} / d=\lambda / N p_{1}$ (see Fig. 1(b)). If $\alpha$ is larger than $\alpha_{c}$, the blurring will fully affect the interference pattern (blurring window larger than one period, $p_{2}$ ). Smaller scattering angles will, on the contrary, have a reduced impact. Recalling that the scattering angle scales with $\lambda / 2 \pi a$, this explains that $(1-\gamma)$ is equal to 1 until $a=N p_{1} / 2$ and then decreases (red dot-dashed curve in Fig. 2(a)). The exact form of the curve depends on the shape of the particles.

Due to the combination of both effects, the SDFI signal reaches a minimum, $V_{\text {min }}$, as plotted in Fig. 2 (blue solid curve in Fig. 2(a)). As can be seen in Fig. 2(b), $V_{\min }$ increases as a function of the energy. For lower energies, the visibility goes to zero, i.e., the interference modulations are completely blurred and the reconstructed SDFI signal will be noisy. ${ }^{12}$ In conclusion, it was seen that SDFI is related to both the particle size and density. Thus, SDFI is a good tool for the investigation of porous materials. Since the length scale of the micro-structure contributing to the signal is not linked to the detector resolution, large samples can be imaged.

\section{EXPERIMENTAL}

The sample, prepared by Audi AG, was produced by Metal Inert Gas (MIG)-welding aluminum/aluminum of two plates placed at a right angle. A photograph can be seen in Fig. 3(a). During the process, pores appear in the weld pool, which are called blow holes. The density and size of the blow holes depend upon the process conditions and partly determine the mechanical resistance of the joint.

The x-ray grating interferometer consists of a standard $\mathrm{x}$-ray tube from Comet (MXR-160HP 20 with focal spot size of $1 \mathrm{~mm}$ according to norm EN 121543) and an x-ray detector from RadIcon (Shad-o-Box 2k) with $2048 \times 1024$ pixels of size $48 \times 48 \mu \mathrm{m}^{2}$ and a scintillator from Kodak (Min-R 2190). The x-ray gratings were optimized for an energy of $50 \mathrm{keV}$ and produced in the clean rooms of the CSEM in Neuchâtel (Switzerland). The periodicities of $G_{0}, G_{1}$, and $G_{2}$ were $20 \mu \mathrm{m}, 4 \mu \mathrm{m}$, and $5 \mu \mathrm{m}$ and the depths were $100 \mu \mathrm{m}$, $32 \mu \mathrm{m}$, and $100 \mu \mathrm{m}$, respectively. The distances were set to $L=1613 \mathrm{~mm}$ and $d=403 \mathrm{~mm}$, which correspond to a Talbot order, $N=1 / 2$. The spatial resolution of the reconstructed images is of the order of $48 \mu \mathrm{m}$.

The two-dimensional radiographies of the attenuation, $T$, and the scatter dark field, $V$, of the sample were reconstructed from a series of 20 phase steps with an exposure time of $6.7 \mathrm{~s}$ at each step. The acceleration voltage of the $\mathrm{x}$-ray tube was set to $70 \mathrm{kVp}$. Computed tomography was also implemented by acquiring 361 projections of the sample by angular steps of $1^{\circ}$ (see Fig. 1(a)). The cone-beam filtered back-projection algorithm developed for phase contrast imaging at Empa ${ }^{14,15}$ was used to reconstruct both the linear absorption coefficient, $\mu$ (from the absorption-based contrast), as along with the

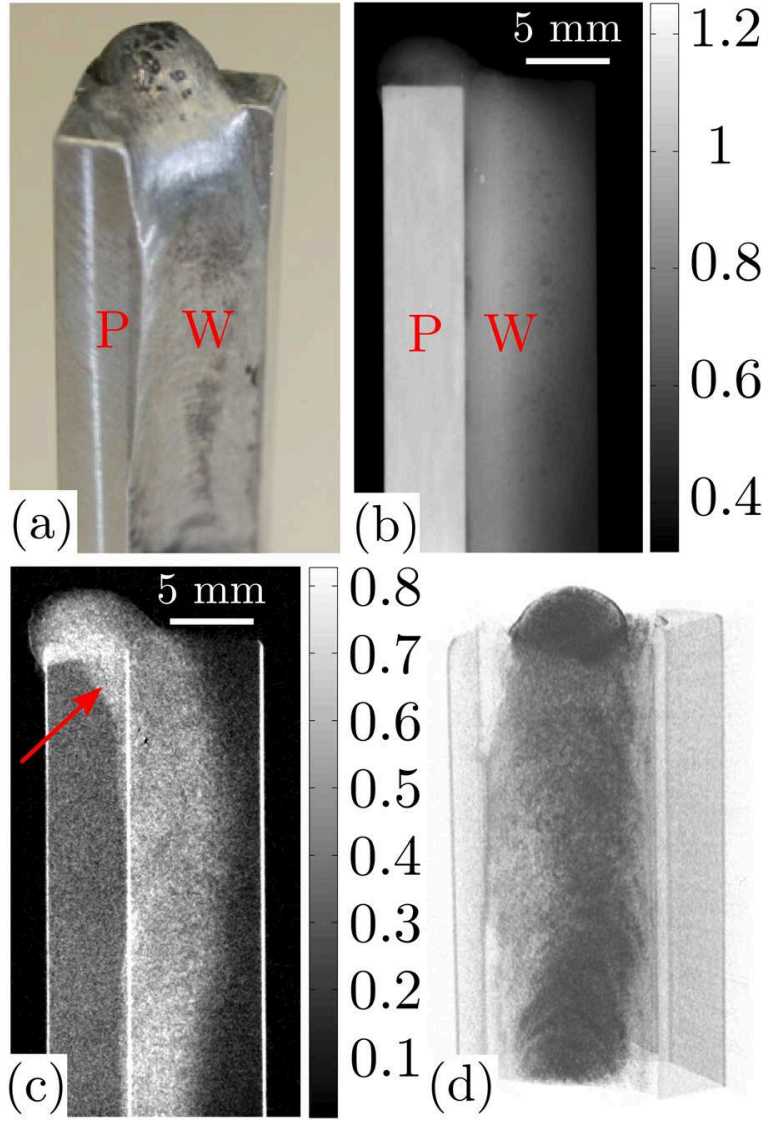

FIG. 3. (Color online) (a) Photograph of the aluminum weld. (b) Logarithms of the absorption-based radiography, $-\log (T)$, and (c) scatter dark field image, $-\log (V)$, of the aluminum weld. The letters, $\mathrm{W}$ and $\mathrm{P}$, have been placed to distinguish the weld from the plates. Note that the weld can be better differentiated from the plates in the scatter dark field image. (d) Threedimensional rendering of the aluminum weld done using the reconstructed linear scattering coefficient.

quantity, $\epsilon=\partial \sigma^{2}(m, n)\left(1-\gamma\left(-N p_{1} ; m, n\right)\right) / \partial z$, which can be interpreted as a linear scattering coefficient. The tomographic reconstruction of the linear scattering coefficient assumes that the inhomogeneities are isotropic. This is justified in the present case, since the blow holes are spherical, which can be verified by looking at the three-dimensional reconstructions of the linear absorption coefficient obtained by micro-tomography.

In order to confirm our results, comparative measurements have been done with an x-ray micro-computed tomography instrument (uCT) located at Empa. ${ }^{16}$ It comprises a $\mu \mathrm{m}$-spot size $\mathrm{x}$-ray tube, a high precision rotation table mounted on a XYZ stage made of three linear stages, and an X-ray flat panel detector with pixels $50 \times 50 \mu \mathrm{m}^{2}$ in size. $2 \times 2$ pixels were binned together for the measurement. The distance between the source and the detector was $500 \mathrm{~mm}$ while the test object was placed at a distance of $50 \mathrm{~mm}$ from the source. The spatial resolution of the reconstructed images is $10 \mu \mathrm{m}$.

The $\mathrm{x}$-ray tube was biased at an acceleration voltage of $70 \mathrm{kVp}$. The 721 projected images, evenly distributed over $360^{\circ}$, were recorded. For each projection, 8 individual images with an integration time of $0.5 \mathrm{~s}$ were recorded. The three-dimensional image of the linear absorption coefficient 
in the test object was calculated by means of a filtered backprojection Feldkamp algorithm. ${ }^{15}$

\section{RESULTS AND DISCUSSION}

Two-dimensional radiographs of the sample are displayed in Figs. 3(b)-(c). A clear contrast improvement can be observed between the absorption-based image, $T$, and the scatter dark field image, $V$. Indeed, while the density of the weld is very similar to the aluminum plates (the same material, hence, a similar absorption), the micro-structures differ. The aluminum plates are essentially homogeneous but the weld is composed of a multitude of blow holes of various sizes. As explained in Sec. II, the difference in USAS then appears clearly in the SDFI signal. Note that the image, $V$, already allows us to observe that the weld pool extends in the aluminum plates, as indicated by the red arrow in Fig. 3(c).

Turning to the results of the computed tomography, an axial slice within the coefficients, $\mu$ and $\varepsilon$, is depicted in Figs. 4(a)-(b). As expected, the value of $\mu$ is identical within the weld and the plates, such that it is hard to distinguish one from the other. Although some large blow holes can be observed, the majority are a size below the spatial resolution of this measurement and thus stay invisible. On the contrary, the cross-section of $\varepsilon$ reveals a clear contrast between the weld and the plates. Compared to two-dimensional projections, the three-dimensional reconstruction enables a precise visualization of the weld volume, notably, to observe the interface between the weld and the plates. Figure 3(d) (Media 1) displays one frame from a movie showing a threedimensional rendering of the weld volume. See the supplementary material for the full movie. ${ }^{17}$

Finally, these results were compared to the three-dimensional reconstruction of $\mu$ obtained with the $\mathrm{uCT}$ instrument. Using VG-studio Max, the data was segmented in order to extract the positions and volumes of the blow holes using the built-in automatic threshold selection. For the same axial cross-section as in Figs. 4(a), and (b), Fig. 4(c) shows the spatial distribution of the blow holes with the volume represented by a color code.

By comparison of the magnified images in Figs. 4(d) and (e), two conclusions can be drawn. First, $\varepsilon$ is close to zero for blow holes that are much larger than the pixel size. Indeed, such holes form a homogeneous area (trapped gas) and do not result in a SDFI signal. Second, the value of $\varepsilon$ appears to be correlated with the density of the holes. The denser the holes, the larger the value of $\varepsilon$, as highlighted, for instance, by the arrows in Fig. 4. Moreover, it can be observed that $\varepsilon$ also takes large values in regions where no blow holes can be observed. In fact, the spatial resolution of the uCT instrument lies at $10 \mu \mathrm{m}$ while the USAS is maximum for inhomogeneities of a size around $2 \mu \mathrm{m}$. Thus, the observed USAS must be related to blow holes of sizes smaller than $10 \mu \mathrm{m}$.

Note that the density of blow holes is lower close to the interface with the aluminum plates. We suppose that this distribution is due to the temperature profile of the workpiece during welding.
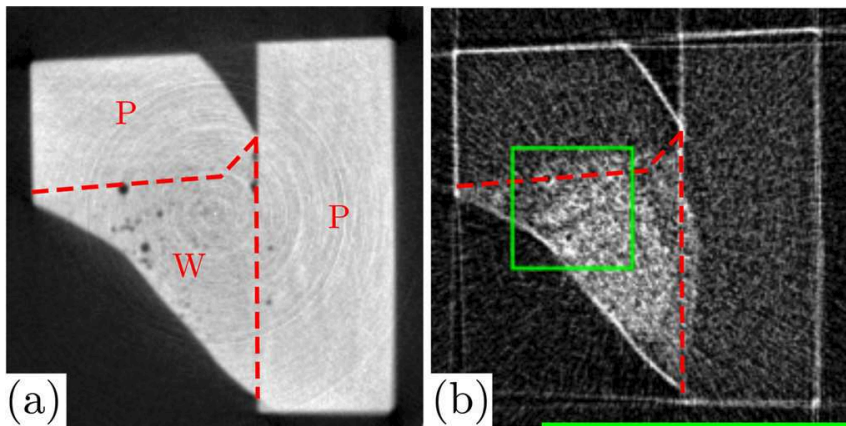

Holes

Volume $\left(10^{3} \times \mu m^{3}\right)$

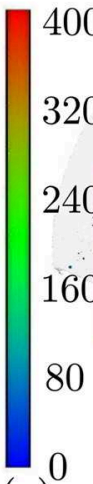

(c)

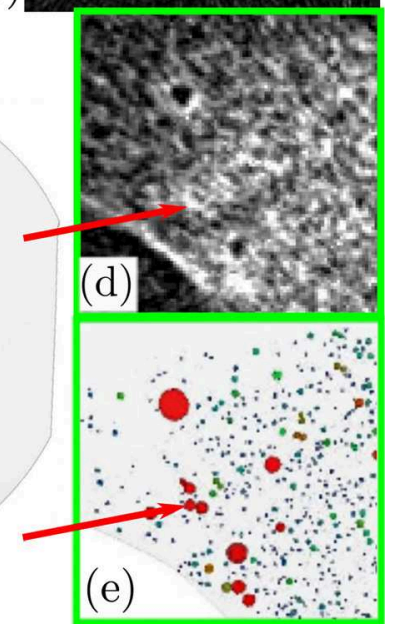

FIG. 4. (Color online) Axial cross sections within (a) the linear absorption coefficient and (b) the linear scattering coefficient reconstructed using the grating interferometer with a spatial resolution $\sim 48 \mu \mathrm{m}$. The letters, $\mathrm{W}$ and $\mathrm{P}$, indicate the positions of the aluminum weld and plates, respectively, and the dashed red lines mark the position of the plate's limits before welding. (c) Visualization of the hole distribution and volume (color coded) from the segmentation of the linear absorption coefficient using the micro-tomography setup with a spatial resolution of $10 \mu \mathrm{m}$. (d) and (e) Close-up images on the areas indicated by the green rectangles in (b) and (c), respectively. As indicated, for instance, by the arrows, the denser the holes, the larger the value of the linear scattering coefficient.

\section{CONCLUSION}

X-ray scatter dark field imaging (SDFI) based on the Talbot-Lau interferometer was used to study porosity at a sub-pixel length scale. The reconstructed SDFI signal is linked to the small angle scattering of the beam in the sample that depends, in particular, on the density and size of the pores. Aluminum welding was used as an example since microscopic gas inclusions, called blow holes, form in the weld during the process. Standard methods for the analysis of such blow holes (for instance, micro-computed tomography) are either time-consuming and/or not adapted to the study of large samples.

For this experiment, a Talbot-Lau interferometer was constructed with a design energy around $50 \mathrm{keV}$ that allows objects made out of light metals to be imaged. Using SDFI, the interface between the weld pool and the workpiece along with the local density of the blow holes within the weld could be unequivocally visualized. Although similar information could also be obtained from a micro-tomographic setup, the proposed method does not require a high spatial resolution and can be achieved with standard x-ray tubes with large focal spot. As a consequence, SDFI can potentially be used for the inspection of large samples within 
shorter exposure times. Moreover, the signal already appears in two-dimensional projections. This represents a decisive advantage in terms of throughput for many industrial applications. For these reasons, we see a great potential for SDFI to become a standard procedure for the inspection of porosity in metals and in injected plastics.

\section{ACKNOWLEDGMENTS}

We would like to acknowledge the work done by our colleagues from the CSEM in Neuchâtel, Francis Cardot and Philippe Niedermann, in the fabrication of the x-ray gratings with high aspect ratios. We would like to thank Audi for providing the aluminum sample. This work was partly financed by the CCMX platform under Grant No. 0206088.

${ }^{1}$ C. David, B. Nöhammer, and H. H. Solak, Appl. Phys. Lett. 81, 3287 (2002).

${ }^{2}$ A. Momose, Opt. Express 11, 2303 (2003).

${ }^{3}$ T. Weitkamp, A. Diaz, C. David, F. Pfeiffer, M. Stampanoni, P. Cloetens, and E. Ziegler, Opt. Express 13, 6296 (2005).

${ }^{4}$ F. Pfeiffer, T. Weitkamp, O. Bunk, and C. David, Nat. Phys. 2, 265 (2006).

${ }^{5}$ F. Pfeiffer, M. Bech, O. Bunk, P. Kraft, E. Eikenberry, C. Brönnimann, C. Grünzweig, and C. David, Nat. Mater. 7, 134 (2008).

${ }^{6}$ W. Yashiro, Y. Terui, K. Kawabata, and A. Momose, Opt. Express 18, 16890 (2010).
${ }^{7}$ V. Revol, C. Kottler, R. Kaufmann, I. Jerjen, T. Lüthi, F. Cardot, P. Niedermann, U. Straumann, U. Sennhauser, and C. Urban, Nucl. Instrum. Methods Phys. Res. A 648, S302 (2011).

${ }^{8}$ F. Pfeiffer, C. Kottler, O. Bunk, and C. David, Phys. Rev. Lett. 98, 108105/1 (2007).

${ }^{9}$ C. Kottler, F. Pfeiffer, O. Bunk, C. Grünzweig, and C. David, Rev. Sci. Instrum.78, 043710 (2007).

${ }^{10}$ C. Kottler, C. David, F. Pfeiffer, and O. Bunk, Opt. Express 15, 1175 (2007).

${ }^{11}$ C. Kottler, F. Pfeiffer, O. Bunk, C. Grünzweig, J. Bruder, R. Kaufmann, L. Tlustos, H. Walt, I. Briod, T. Weitkamp, and C. David, Phys. Status Solidi A 204, 2728 (2007).

${ }^{12}$ V. Revol, C. Kottler, R. Kaufmann, U. Straumann, and C. Urban, Rev. Sci. Instrum. 81, 073709 (2010).

${ }^{13}$ V. Revol, C. Kottler, R. Kaufmann, F. Cardot, P. Niedermann, I. Jerjen, T. Lüthi, U. Straumann, U. Sennhauser, and C. Urban, "Sensing Small Angle Scattering with an X-ray Grating Interferometer," Nuclear Science Symposium Conference Record (NSS/MIC), Knoxville USA, Oct. 30 - Nov. 06, (2010).

${ }^{14}$ I. Jerjen, V. Revol, C. Kottler, R. Kaufmann, T. Lüthi, and U. Sennhauser, Proc Int. Conf. Adv. Phase Meas. Meth. Opt. Imaging, 1236, 227 (2010).

${ }^{15}$ L. A. Feldkamp, L. C. Davis, and J. W. Kress, J. Opt. Soc. Am. A 1, 612 (1984).

${ }^{16}$ P. Trtik, B. Münch, W. J. Weiss, A. Kaestner, I. Jerjen, L. Josic, E. Lehmann, and P. Lura, "Release of internal curing water from lightweight aggregates in cement paste investigated by neutron and X-ray tomography," Nucl. Instrum. Meth. Phys. Res. A, in press, 2010.

${ }^{17}$ See supplementary material at http://dx.doi.org/10.1063/1.3624592 for a movie showing a three-dimensional rendering of the weld volume. 